\title{
Rome, Season Two Episode Listing
}

Episode I3, "Passover," written by Bruno Heller, directed by Tim van Patten

Episode I4, "Son of Hades," written by Bruno Heller, directed by Allen Coulter

Episode I 5, "These Being the Words of Marcus Tullius Cicero," written by Scott Buck, directed by Alan Poul

Episode I6, "Testudo et Lepus (The Tortoise and the Hare)," written by Todd Ellis Kessler, directed by Adam Davidson

Episode I7, "Heroes of the Republic," written by Mere Smith, directed by Alik Sakharov

Episode I 8, "Philippi," written by Eoghan Mahony, directed by Roger Young

Episode I9, "Death Mask," written by Scott Buck, directed by John Maybury

Episode 20, "A Necessary Fiction," written by Todd Ellis Kessler, directed by Carl Franklin

Episode 2I, "Deus Impeditio Esuritori Nullus (No God Can Stop a Hungry Man)," written by Mere Smith, directed by Steve Shill Episode 22, "De Patre Vostro (About Your Father)," written by Bruno Heller, directed by John Maybury 